\title{
Properties of Retinal Induction in the Human Eye
}

\author{
By \\ Koiti Motokawa \\ (本川 弘一) \\ (From the Physiological Laboratory, Tohoku \\ University, Sendai, Japan) \\ (Received for publication, June 23, 1952)
}

\section{INTRODUCTION}

Visual contrast or induction is a familiar phenomenon, but its mechanism is quite obscure. It must be due to the lack of adequate methods to make this phenomenon accessible to any quantitative measurements.

In the previous papers ${ }^{12)}$ it was shown by the author that the method of electro-stimulation, which had proved to be a serviceable means for analyzing retinal color processes, could be used for the study of retinal induction as well. Some properties of retinal induction were revealed by this method, and the importance of retinal induction as a physiological event underlying a variety of psychological phenomena such as contrast, optical illusion, "gestalt", etc. was emphasized based upon the data obtained by this method.

However, physiological data concerning; this phenomenon are yet so scant that we have yet no clue to the mechanism of induction. What we can do at present is to provide experimental materials as many as possible and to try to find any regularities and interrelations existing among them.

\section{EXPERIMENTAL}

\section{Method}

In the preceding paper,") the terms "direct induction" and "indirect induction" were introduced in order to distinguish physiological effects from psychological ones such as successive and simultaneous induction. By direct induction we mean a physiological after-effect due to an illumination which is proved within the area pre-illuminated, and by indirect induction a physiological effect simultaneously induced around a retinal image is meant. In the present investigation these terminologies 
will be used, and the effect of light will be expressed in terms of $\zeta$ which is defined by the formula: $\zeta=100\left(E-E_{0}\right) / E_{0}$, where $E$ and $E_{\circ}$ denote electrical sensitivities measured with and without pre-illumination.

As inducing light exclusively sodium yellow light was used, for such strong monochromatic light was advantageous for producing strong retinal induction. To measure the direct induction caused by the sodium yellow light, the electrical excitability is measured at the moment 3 seconds after termination of white test-light which is preceded by the yellow inducing light. $\zeta$-values determined in this way are generally higher than those for the white test-light alone. The difference of these two kinds of $\zeta$-values is termed "contrast effect" and serves as a quantitative measure for retinal induction.

In order to measure indirect induction, the subsequent white testlight is made to fall on an adjacent part to the retinal area pre-illuminated by the yellow light and excitability measurements are done $1.5 \mathrm{sec}$. instead of $3 \mathrm{sec}$. after removal of the white light. As to the technique of electrostimulation of the eye the papers ${ }^{3 / 4}$ should be consulted.

The patches used for illumination consisted of a plate of ground glass and 2 sheets of black paper. An example is shown in Fig. 1. In this case the inducing figure is a half-ring (A), and the white test-light is a circular patch $2 \mathrm{~mm}$. in diameter (B). The latter is to be presented

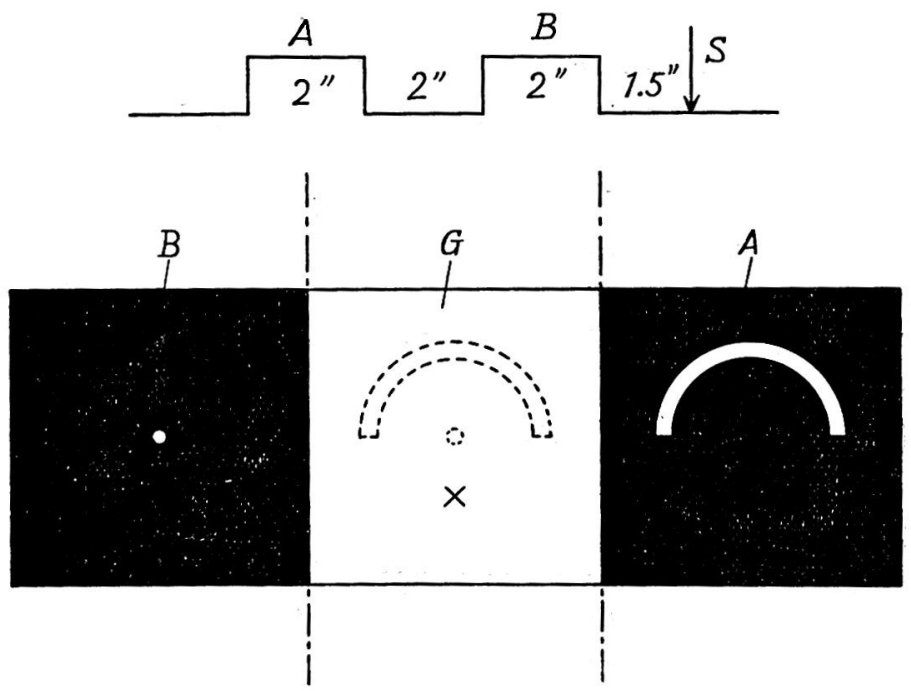

Fig. 1. Arrangement of patches. A: a sheet of black paper on which an inducing figure is cut (a half-ring in this case). B: a sheet of black paper with a circular opening of $2 \mathrm{~mm}$. in diameter. G: ground glass. Order of presentation of patches is indicated on top. 
at the center of the half-ring. When the inducing figure was to be presented, the paper A was laid upon the ground glass, and then this was illuminated by the yellow light from behind. To present the white testlight, the ground glass was illuminated by white light after the paper A had been replaced by the paper B. The operation of these patches $A$ and $B$ was done by the subject in the dark interval between successive illuminations. The distance between the patch and the subject's eye was always $30 \mathrm{~cm}$.

\section{Results}

1. Dependence of retinal induction upon retinal area illuminated

In the previous experiment ${ }^{1}$ it was established that the intensity of retinal induction expressed in terms of contrast effect is proportional to the logarithm of the intensity of inducing light, while it depends little on the intensity of the white test-light. The question now arises as to how it depends on the area illuminated.

In the following experiment, the direct induction caused by circular patches of varying diameters was measured, using white test-patches of the same size as the inducing ones. The data are shown in Table I. As

TABLE I

Illuminated Area and Intensity of Direct Induction

\begin{tabular}{c|c|c|c|c}
\hline $\begin{array}{c}\text { Diameter of patch } \\
\text { in mm. }\end{array}$ & $\zeta$ for yellow alone & $\begin{array}{c}\zeta \text { for white } \\
\text { alone }\end{array}$ & $\begin{array}{c}\zeta \text { for yellow- } \\
\text { white }\end{array}$ & Contrast effect \\
\hline 2 & 24.5 & 19.5 & 28.5 & 9.0 \\
4 & 30.0 & 25.0 & 45.5 & 20.5 \\
8 & 46.0 & 41.0 & 61.3 & 20.3 \\
16 & 48.5 & 43.5 & 64.0 & 20.5
\end{tabular}

can be seen in this table, $\zeta$-values for the yellow patch alone or the white patch alone increase with their diameter. This is an expression of spatial summation of retinal excitation. The contrast effect also increases with the area so long as it is sufficiently small, but remains almost constant irrespective of the area illuminated when it is greater than $3 \mathrm{~mm}$. It is to be noted that summation is seen with respect to retinal excitation or $\zeta$-values for yellow or white illumination alone, whereas no summation is found with respect to induction or contrast effects.

Next, the dependence of indirect induction upon the area illuminated was studied, using rings of varying widths as inducing patches. The white test patch used was a circle of $2 \mathrm{~mm}$. in diameter presented at the center of the ring (see Fig. 2). The data obtained are represented in 

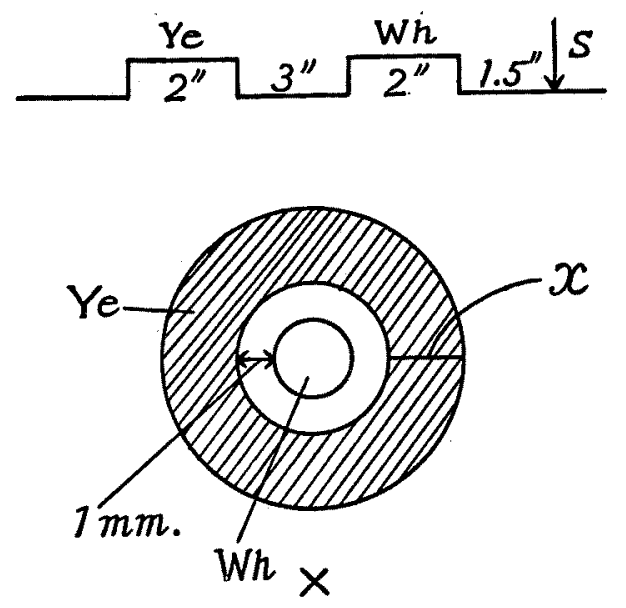

Fig. 2. Ye: yellow inducing patch. Wh: white test-patch. $\mathbf{x}$ : variable. Cross indicates point of fixation.

Table II. Contrast effects were found almost constant, indicating that indirect induction is independent of the width of the inducing ring. From this finding it follows that the indirect induction at the center of the ring

TABLE II

Illuminated Area and Intensity of Indirect Induction

\begin{tabular}{c|c|c|c}
\hline $\begin{array}{c}\text { Width of ring } \\
\text { in Fig. 2 }\end{array}$ & $\zeta$ for white alone & $\zeta$ for yellow-white & Contrast effect \\
\hline $1 \mathrm{~mm}$. & 26 & 46.8 & 20.8 \\
$2 "$ & $"$ & 46.0 & 20.0 \\
$4 "$ & 45.2 & 19.2 \\
$8 "$ & & 46.0 & 20.0
\end{tabular}

was determined by the light falling within the innermost zone of about $1 \mathrm{~mm}$. width of the ring, while the other part of the ring contributed nothing to the induction under consideration.

Another series of experiment was carried out with a rectangular patch $20 \mathrm{~mm}$. long. The width of the rectangle was varied and the indirect induction at a point $3 \mathrm{~mm}$. from the margin was measured. The data are shown in Table III, which indicates that the contrast effect is independent of the area of the rectangle when its width is greater than $2 \mathrm{~mm}$. This finding also suggests that the area itself plays no important role in producing retinal induction. In view of the phenomenon of border contrast it is conceivable that the contour of an inducing figure plays a more prominent role in this regard, and the following experiment seems 

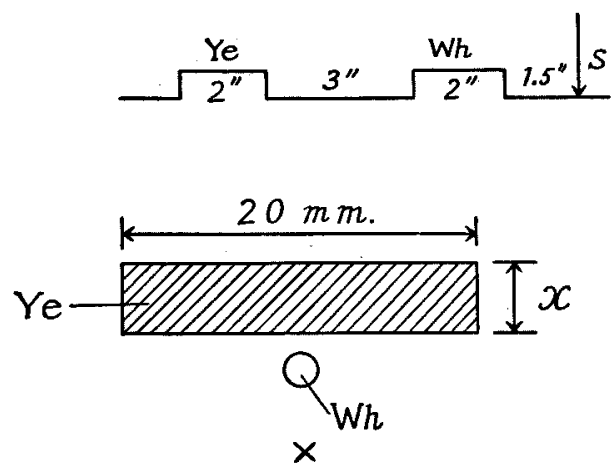

Fig. 3. All notations as in Fig. 2.

\section{TABLE III}

Illuminated Area and Intensity of Indirect Induction

\begin{tabular}{c|c|c|c}
\hline $\begin{array}{c}\text { Width in mm. of } \\
\text { rectangle in Fig. 3 }\end{array}$ & $\zeta$ for white alone & $\zeta$ for yellow-white & Contrast effect \\
\hline 1.0 & 21 & 31 & 10 \\
2.0 & $"$ & 42 & 21 \\
3.5 & $"$ & 43 & 22 \\
4.0 & " & 43 & 22 \\
8.0 & 42 & 21
\end{tabular}

to support this view. The field of retinal induction around a single horizontal bar of $20 \mathrm{~mm}$. in length and $2 \mathrm{~mm}$. in width was compared with the field set up by a horizontal broken bar of the same length and witdh. In this experiment the contrast effect at any point of the field was found greater for the broken bar than for the continuous one; the field around the continous bar extended over a distance of about $17 \mathrm{~mm}$. in the direction perpendicular to the bar, while with the broken bar the corresponding distance was found to be about $31 \mathrm{~mm}$. The greater extension of the field around the broken bar may be ascribed to its longer contour, but the possibility cannot be ruled out that other factors might be responsible for such a great difference of indirect induction caused by the continuous and the broken bars.

2. Summation of indirect induction

The arrangement of patches used in the following experiment is shown in. Fig. 4. The contrast effect at the center of the half-ring was found to be 11 , while the value at the center of the broken ring was as high as 27. The two inducing figures used here were the same in area, but somewhat different in the length of contour; the contour of the half- 


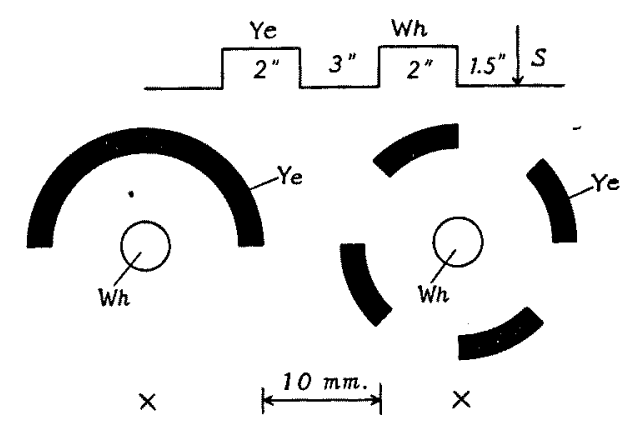

Fig. 4. All notations as in Fig. 2.

ring was $60.26 \mathrm{~mm}$. while that of the broken ring was $72.26 \mathrm{~mm}$. Although it cannot be denied that the contour is one of the most important factors for establishing indirect induction, the difference in the length of contour seems to be too small to account for the great difference in contrast effects in this case. It seems as if the high effectiveness of the broken ring is due to its configuration advantageous for summation of indirect induction. Generally, the value of indirect induction is found greater within a closed line figure than outside it. This fact shows that summation takes place more easily at a point enclosed by inducing sources. In the case of the half-ring the distribution of inducing sources is less enclosing than in the case of the broken ring, so that the summation of indirect induction must have accordingly been slight.

In order to study the effect of summation under simpler experimental conditions, the contrast effect was measured at a point midway between 2 vertical bars $20 \mathrm{~mm}$. in length and $2 \mathrm{~mm}$. in width and compared with the contrast effect obtained with a single bar. In Fig. 5 the values of contrast effects are represented as a function of distance from the margin of the bar. Though the area and contour of the double figure are twice as large as the single, the contrast effect in the former is not always twice as that in the latter; the ratio of the two values varies remarkably with the distance from the margin. These findings indicate that the indirect induction must be a very complicated function of distance, contour, configuration and other yet unknown factors.

3. Dependence of indirect induction on angles of inducing figures

The patch used in the following experiment is shown in the inset of Fig. 6. The effect of angle $\theta$ upon the contrast effect measured at point Wh was investigated as a function of $\theta$. The contrast effect decreased as the angle $\theta$ increased and reached a minimum at $90^{\circ}$. It is apparent that the contour, area and distance remained almost unaltered in this experiment. In spite of this, the contrast effect greatly changed 


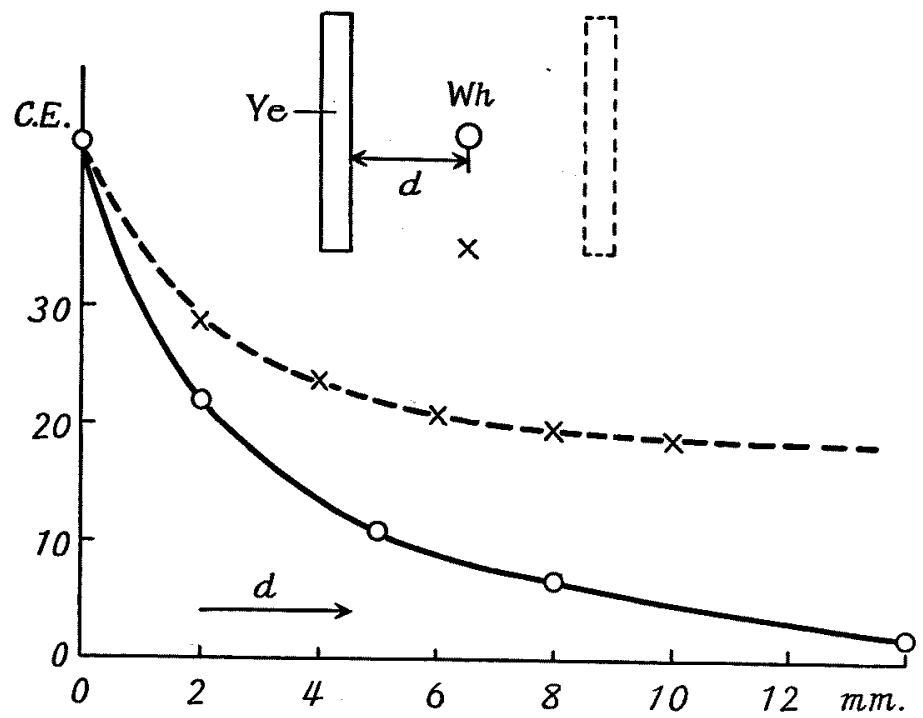

Fig. 5. Dependence of contrast effect (C.E.) on distance from margin of inducing yellow bar $20 \mathrm{~mm}$. long and $2 \mathrm{~mm}$. wide. Broken and continuous curves refer to induction caused by 2 bars and to that caused by a single bar respectively.

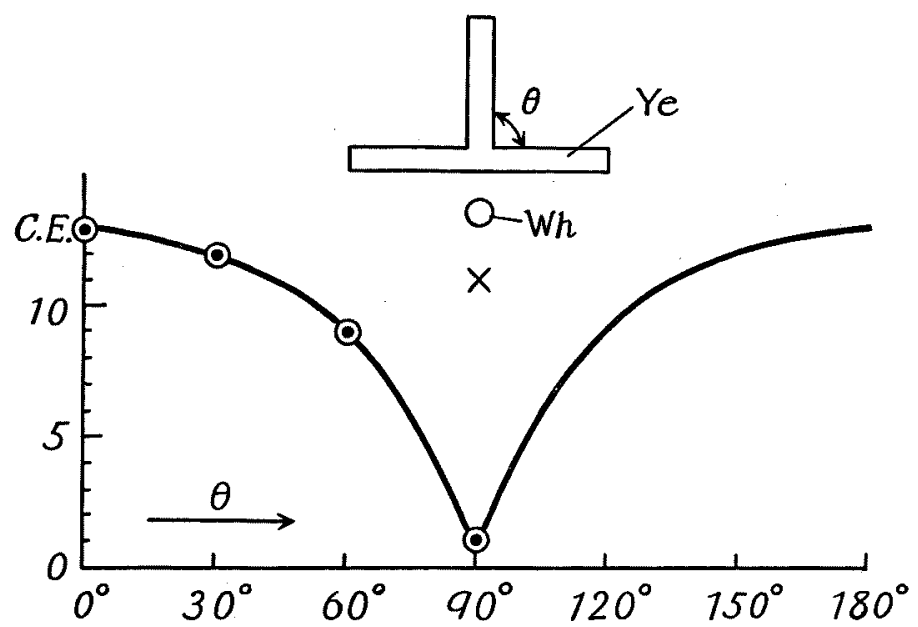

Fig. 6. Dependence of C.E. on angle.

depending on the angle $\theta$. This fact indicates that the angle as such" is an important factor to set up indirect induction.

Next, the inducing figure was so modified that the horizontal bar 


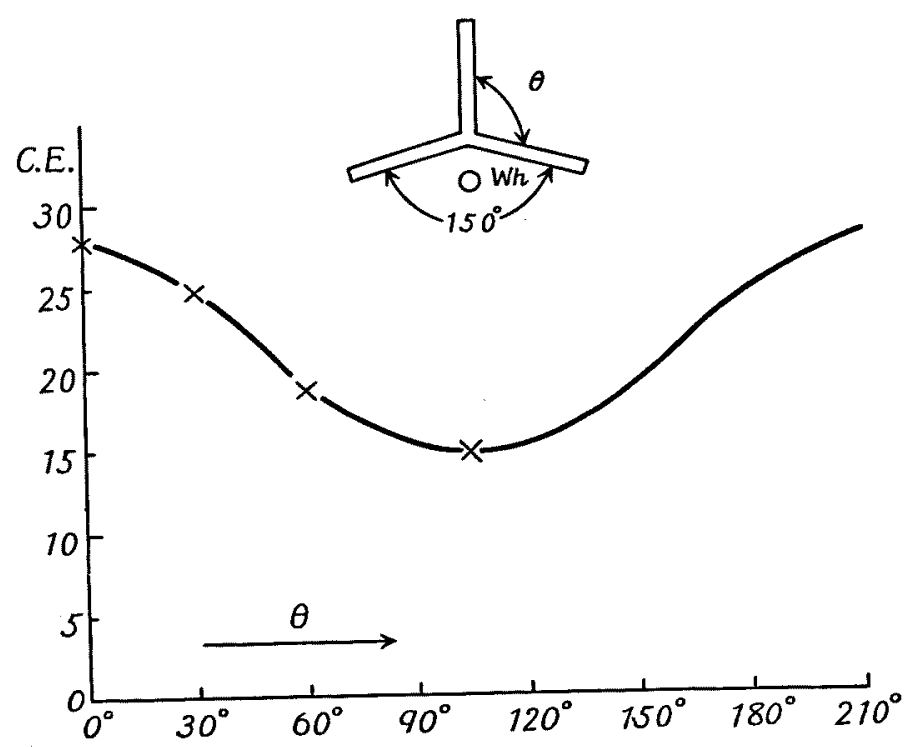

Fig. 7. Dependence of C.E. on angle.

was no more a straight line but consisting of 2 bars meeting at $150^{\circ}$, the other features being the same as in the experiment stated above. The contrast effect showed remarkable dependence on the angle $\theta$ in this experiment, too. It decreased with increasing $\theta$ and reached a minimum in the vertical position of the additional bar. The difference found between the two experiments was that the minimum value of contrast effect was much lower for the figure with the straight horizontal bar. The angle for the minimum of contrast effect was $90^{\circ}$ in the one case and $105^{\circ}$ in the other. This difference must be responsible for the difference mentioned above, for it was established in the preceding paper ${ }^{2}$ that a right angle or its multiples are more effective for reducing the intensity of indirect induction than other angles.

\section{SUMMARY}

Retinal induction in the human eye was measured by the method of electro-stimulation to investigate its dependence on various properties of the inducing figure such as area, contour, configuration, angle, etc.

1. Direct induction by which a physiological effect remaining within the area pre-illuminated is meant depends little on the area of the retinal image unless it is too small.

2. The same is true for indirect induction set up simultaneously around a retinal image; for example, the indirect induction set up by 
a half-ring at its center was found much weaker than that set up by a broken ring of the same diameter, although the area illuminated was the same in the 2 cases.

3. Indirect induction becomes weaker as the distance from the margin of the inducing figure increases. The gradient of induction is, however, less steep when more than one inducing source are present (summation).

4. Angles contained in an inducing figure are an effective factor for producing indirect induction. This relation was illustrated by two examples.

\section{References}

1) Motokawa, K., J. Neurophysiol., 1949, 12, 475.

2) Motokawa, K., J. Neurophysiol., 1950, 13, 413.

3) Motokawa, K., J. Neurophysiol., 1949, 12, 291.

4) Motokawa, K., Tohoku J. Exp. Med., 1951, 54, 385. 